\title{
Leszek Ploch
}

ORCID: 0000-0002-3759-5445

Uniwersytet Przyrodniczo-Humanistyczny w Siedlcach

Wydział Nauk Społecznych

\section{Initiativen zur sozialen Eingliederung und Forschung zur Verbesserung der Lebensqualität der Menschen mit Behinderungen in Deutschland}

\author{
Social integration initiatives and research \\ to improve the quality of life of persons with disabilities \\ in Germany
}

https://doi.org/10.34739/sn.2019.19.01

\begin{abstract}
Kurzfassung: Der vollen Ideen und Forschung Sozialen Arbeit im Kontext Mensch mit Behinderung in Deutschland orientiert sich nicht nur nach Inklusion aber auch nach einem ursprünglichen schulpädagogischen Diskurs, der jedoch spätestens mit der Ratifizierung der UN-Behindertenrechtskonvention (UB-BRK) im Jahr 2009 in nahezu allen Bereichen der Pädagogik den Fachdiskurs maßgeblich prägt. Ein wichtiges außerschulisches pädagogisches Handlungsfeld, das sich die Verwirklichung einer „inklusiven Gesellschaft" zur Aufgabe gemacht hat, ist die sozial-kulturelle Inklusion und Bildung explizit erwähnt. Immer wieder Zukünftig gehe es darum, Menschen mit Behinderungen barrierefreie Zugänge zu den kulturellen Materialien und Orten des Sozial-kulturelles Leben zu ermöglichen.
\end{abstract}

Schlüsselwörter: Mensch mit Behinderung, Sozialarbeit, Inklusion, Forschung

\begin{abstract}
The ideas and research in the field of social work in the context of people with disabilities in Germany are based not only on integration activities, but also on pedagogical discourse. Ratified in 2009 the UN Convention on the Rights of Persons with Disabilities (CRPD) defined specialist scientific discourse in almost all areas of pedagogy and social work. An important non-school field of pedagogical activities, which has made the implementation of 'inclusive society' its task, is to include people with disability in social and cultural life of their environment. The aim of such scientific and social work is to provide disabled people with barrier-free access to social activities, but also to culture and cultural products.
\end{abstract}

Keywords: people with disabilities, social work, inclusion, research 
Es soll allen bekannt worden sein, dass Rehabilitation und Eingliederung mehr Fach geistig behinderter und von Behinderung bedrohter Menschen dem Qualitätsanspruch, der an diese Aufgaben gestellt wird, und den Erwartungen der Betroffenen entsprechen, müssen bei ihrer Konzeption und ihrer Durchführung in Deutschland alle praktischen, vor allem aber auch einschlägige wissenschaftliche Erkenntnisse genutzt werden. Das soll bedeuten, dass Entwicklungen in diesem Bereich haben immer mehr Chans vielfach durch Forschungsarbeiten eingeleitet, begleitet, gefördert und dokumentiert und auch sei es durch Einsatz bereits gewonnener Erkenntnisse für diese Aufgaben, sei es durch Forschungs- und Entwicklungsarbeiten mit dem ausdrücklichen Ziel, Sozialrehabilitation, Therapie und Eingliederung mehr Fach Menschen mit Beeinträchtigungen zu verbessern ${ }^{1}$. Anlass für Fortentwicklungen sind teilweise veränderte Anforderungen, wie sie sich z.B. aus „neuen Technologien“, aus dem Bemühen um „neue“ Behindertengruppen wie den Aspergera-Syndrom oder aus „neuen“ Erfordernissen von Wirtschaftlichkeit und herführten Inklusion Prozess ergeben. Die unterschiedlicher SozialKulturellen Initiativen und geführte Forschungsarbeiten in Therapie Bereichen haben daher großen Einfluss auf Ziele und Wege der Rehabilitation und der Eingliederung in Inklusion Zone die Menschen mit Beeinträchtigungen [Boban, Hinz, 2010].

Für 2017 ist im Rahmen einer Gesetzesreform (Bundesteilhabegesetz) eine Neufassung von Definition Behinderung entwickeln. Sie nach dem Stand der Entwurfsfassung vom 26. April 2016 wie folgt lautet: »Menschen mit Behinderungen sind Menschen, die körperliche, seelische, geistige oder Sinnesbeeinträchtigungen haben, welche sie in Wechselwirkung mit einstellungs- und umweltbedingten Barrieren an der gleichberechtigten Teilhabe an der Gesellschaft mit hoher Wahrscheinlichkeit länger als sechs Monate hindern können. Eine Beeinträchtigung nach Satz 1liegt vor, wenn der Körperund Gesundheitszustand von dem für das Lebensalter typischen Zustand abweicht. «Wenn es bei dieser Formulierung bleibt, wird sich im Ergebnis und den rechtlichen Konsequenzen wenig verändern. Die Definition ist insgesamt

\footnotetext{
${ }^{1}$ Man sollte daran hinweisen das, in der Bundesrepublik Deutschland der leistungsrechtlich relevante und in Fachkreisen und Bevölkerung häufig verwendete Begriff "geistige Behinderung" wird von Menschen mit kognitiven Beeinträchtigungen als diskriminierend erlebt. Sie nennen sich selbst Menschen mit Lernschwierigkeiten und fordern die Abschaffung des Begriffs geistige Behinderung (vgl. www.people1.de). Darum wird zur Kennzeichnung des Personenkreises in diesem Bericht überwiegend die Formulierung „kognitive Beeinträchtigung" verwendet.
} 
eher umständlicher, aber deshalb nicht präziser wie ihre Vorgängerin. Auch das Kriterium für Schwerbehinderung (GdB von 50) wird demnach in gleicher Weise gefasst wie vorher. Daraus wird zweierlei deutlich. Zum einen unterscheidet die juristische Definition von Behinderung und damit auch die Schwerbehindertenstatistik nur über ein sehr formales zeitliches Kriterium zwischen krankheits- und behinderungsbedingten Beeinträchtigungen [Kast, 2017, s. 39].

Heute zu Tag die Deutschen Wissenschaftler unterstützten intensiv in Bereich Inklusion verschiedenen Schwerpunktbereichen Forschungs- und Entwicklungsarbeiten mit dem Ziel, die Lebensbedingungen Menschen mit Beeinträchtigungen zu verbessern. Diese geschieht vor allem im Rahmen von Forschungsprogrammen, insbesondere der Programme, z.B.: „Projekt Berufliche Orientierung Inklusion", "Gemeinsam selbstbestimmt leben“, "Werkstätten für Behinderten“, „Neue Fachkräfte auf der Kariere“, „Innovationen auf der Werkstätten“, „Zurück ins Glück - Förderung von Wohnraum für behinderte Menschen“, "Kulturelle Teilhabe von Menschen mit Behinderung", "Gesundheitsforschung 2018" und „Arbeit und Technik", „Beschäftigung und Integration", darüber hinaus werden für spezifische Fragen Behinderter Lösungen durch wissenschaftliche Untersuchungen der dafür zuständigen Ministerien angestrebt [Teilhabebericht der Bundesregierung..., 2013].

Weitere Forschungsimpulse und Sozial-Kulturellen Initiativen werden auch durch die Anregungen der Institutionen, Therapie und Bildung Einrichtungen und Träger sowie durch neue infrastrukturelle Entwicklungen und Wissenschaftlichen Empfehlungen erweitert. Die oben genannten Programme haben einen gemeinsamen Ziel „Forschung und Entwicklung im Dienste der Gesundheit, Therapie, Sozialen und Inklusion Qualität bleiben“ vom einem umfassenden Rehabilitationsverständnis aus, das neben medizinischen auch berufliche und soziale Aspekte einbezieht. Das bedeutet, dass Forschung Schwerpunkte und Themen in Bereich Inklusion reichen von der Diagnostik über Fragen der Behandlung und Rückfallprophylaxe bis zur Gestaltung rehabilitativer und therapeutischer Maßnahmen einschließlich der Entwicklung technischer Hilfen für Menschen mit Beeinträchtigungen. Die Programme sind auf forschungsstrukturelle Wirkungen in sozialpolitisch vorrangigen Forschungsfeldern ausgerichtet. Diese Ausrichtung bietet Möglichkeiten, auch die für eine dauerhafte Verankerung der Rehabilitations- und Therapiewissenschaften, auch Inklusion geeignet Forschungsstrukturen in Universitäten zu entwickeln [Kast, 2017, Meis, Mies, 2012]. 
Die Rehabilitationsträger, Einrichtungen für Bildung und Therapie Maßnahmen Instituten haben in letzten Jahren in eigenen Forschungsarbeiten besonders thematisiert:

- ob die mit medizinischen und sozialen Maßnehmen zur SozialKulturellen Inklusion angestrebten Ziele (z.B. vorzeitige Minderung der Erwerbsfähigkeit zu vermeiden oder zumindest hinauszuzögern) erreicht werden;

- $\quad$ wie die Inanspruchnahme Sozial-Kultureller Leistungen zielgereichtet gesteuert werden kann.

Vielen deutschen Wissenschaftler, Pädagogen und Therapeuten fördern wissenschaftliche Untersuchungen zur Weiterentwicklung der Inklusionsleistungen Prozess und legen dabei seinen Schwerpunkt auf die anwendungsorientierte Forschung. In ihren Vorschlägen zur stärkeren Förderung der Sozial-Kulturellen Inklusion wendet sich die Einrichtungen von Therapie-, Rehabilitation-, Edukation- und Forschungsabteilungen im Verbund mit Universitäten in ausgewellten Rehabilitationseirichtungen selbst. Zum einen betrifft die empfohlene Fortentwicklung ein auf die Erkenntnisinteressen der Inklusionsträger abgestelltes Forschungsprogramm, für das ein Anteil der Aufwendungen für Inklusion von den Inklusionsträgern bereitgestellt werden sollte. Zum anderen die Wissenschaftler, Pädagogen und Therapeuten, die Inklusionswissenschaften in den universitären Forschungsstrukturen so zu verankern, dass eine kontinuierliche inklusionswissenschaftliche Forschung möglich wird.

Aufgrund der zunehmenden Bedeutung der Inklusion Prozess unter Menschen mit Behinderung die Landesregierungen haben zugestimmt, das Bewusstsein der Bevölkerung für jede Form von Diskriminierung zu schärfen und echte Gleichbehandlung herzustellen. Die Landesregierungen wenden sich gegen jede Form von Diskriminierung. Sie verfolgen das Ziel, dauerhafte Grundlagen für ein vorurteils- und diskriminierungsfreies Miteinander zu legen [Amrhein, 2001; Boban, Hinz, 2012, s.22; Übereinkommen der Vereinten Nationen, 2011; Eberwein, Mand, 2008; Hinz, Körner, Niehoff, 2012; Klemm, Preuss-Lausitz, 2011].

Darüber hinaus strebten die Wissenschaftler, Pädagogen und Therapeuten für Arbeit und Sozialen Ordnung an, im Rahmen des Modellprogramms zur Verbesserung der Versorgung der Menschen mit Behinderungen die Entführung und die Umsetzung der Inklusion Prozess durch beispielhafte Vorhaben vorzubereiten und zu begleiten, insbesondere durch: 
- den Ausbau der inklusiver Sozial-Kulturellen Infrastruktur,

- die Verbesserung der Zusammenarbeit aller an der Pädagogik, Therapie und Rehabilitation Beteiligten,

- Fortbildung des Pädagogischen, Therapeutischen und anderen Personals zur aktivierender Sozial-Kulturellen und Inklusion Arbeit,

- Behebung spezifischer Inklusionsdefizite, z.B. durch Kulturelle, musikalische und andere Therapie formen Aktivitäten Menschen mi Behinderungen in den Schulen und sondern Einrichtungen,

- Beratung der Angehörigen für die Nutzung der Einrichtungen und für die Wahrnehmung ihrer Inklusionsrechtlichen Ansprüche.

Da die Inklusion hat sich zu einer dominierenden Leitvorstellung pädagogischer Handlungsfelder entwickelt, das Modellprogramm in Beriecht der Sozial-Kulturellen Inklusion immer wieder unterstütz unter anderem auch die Integration Lebenstherapie Hilfen in die Gesamtversorgung durch ambulante, teilstationäre und stationäre Dienste und Einrichtungen für Menschen mit Beeinträchtigungen. In rahmen Inklusion Arbeit die Bundesregierung unterstütz Forderungen z.B. des Deutschen Vereins für öffentliche und private Fürsorge, die sich vor allem an die Länder, Leistungsträger und Verbände richten und den Aufbau eines fachqualifizierten, regelhaft verfügbaren Therapieangebots für Menschen mit Behinderungen in einem überschaubaren Zeitraum zum Ziel haben. Darüber hinaus zukünftig gehe es darum, Menschen mit Beeinträchtigungen barrierefreie Zugänge zu den kulturellen Materialien und Orten des sozialen Lebens zu ermöglichen [Kröger, Merkt, Sievers, 2014]. Sozial-kulturelles Arbeit ist dabei in den Mittelpunkt gestellt, weil ein großes Interesse an der Umsetzung von Inklusion besteht und nicht zuletzt auch, weil jahrelange Praxiserfahrung in diesem Feld verfügt und eine Expertise einbringen kann. Vor allem im Feld der Sozialen Arbeit wird inzwischen Wert darauf gelegt kulturpädagogische Angebote inklusiv zu gestalten [Dannenbeck, 2015, s. 15-25; Sauer, 2014]. Inklusive gestaltete Arte-Therapieangebote seien, wie behauptet C. Dorrance, W. Meyberg (2015) in besondere Weise geeignet: „den Bedürfnissen aller Beteiligten, unabhängig von Lernschwierigkeiten, Sinneseinschränkungen oder körperlich begrenzten Möglichkeiten zu entsprechen und über bestehende oder behauptete kulturelle und soziale Grenzen hinweg zur gemeinsamen Interaktion [...] und zu wechselseitiger Kooperation auf demokratischer Basis zu ermutigen“ [Dorrance, Mayberg, 2015, s. 202].

Unter dem deutschen Wissenschaftler zur Diskurs immer wieder häufig kommt Kritik über Stigmatisierungen von Menschen in verschiedenen 
Gruppen, die nicht zum Abbau, sondern zur Reproduktion gesellschaftlicher Marginalisierung beitragen, weil die betroffenen Kinder und Jugendlichen schon früh als „anders" und „leistungsschwach" etikettiert werden. Gruppenbezogene Zuschreibungen gehen häufig einher mit komplexitätsreduzierenden Konstruktionen von Homogenität und führen dazu, dass die Individualität einer Person ignoriert wird oder im Verbogenen bleibt [Boban, Hinz, Plate, Tiedeken, 2014, s. 20]. In der Folge, wie behaupten die Wissenschaftler, kümmern sich Sonderpädagoge ausschließlich um stigmatisierte Schüler und es bleibt bei exklusiven Zuständigkeiten. Zwar werden den Schüler zusätzliche Ressourcen zugewiesen, jedoch nur zum Zweck einer „kompensatorischen Unterstützung zur Wiedergewinnung des Anschlusses an das allgemeine Niveau" [Boban, Hinz, 2012, s. 70].

Im Kontext inklusiver Pädagogik sind institutionelle Etikettierungsprozesse grundsätzlich abzulehnen. Nach der Meinung von Wissenschaftler Inklusion ist nur verwirklicht, wenn auf jegliches Etikettieren bestimmter Gruppen verzichtet wird, um: „alle Dimensionen von Heterogenität in den Blick zu bekommen und gemeinsam zu betrachten. Dabei kann es um unterschiedliche Fähigkeiten, Geschlechterrollen, ethnische Herkünfte, Nationalitäten, Rassen, soziale Milieus, Religionen und weltanschauliche Orientierungen, körperliche Bedingungen, oder anderes gehen. Charakteristisch ist dabei, dass Inklusion sich gegen dichotome Vorstellungen wendet, die jeweils zwei Kategorien konstruieren: Deutsche und Ausländer, Männer und Frauen; Behinderte und Nichtbehinderte, Reiche und Arme usw" [Hinz, 2012, s. 33].

Es ist gut zu Wiesen, dass in den demokratischen Schulen, im Bericht Inklusion erfüllen einige Aufgabe, das staatliche Schulwesen zu ergänzen, zu bereichern und innovative Formen des Unterrichts und der Erziehung zu entwickeln. Privatschulen proklamieren, dass die individuellen Interessen und Lernwege der Schüler ein vielfältiges Bildungsangebot erfordern. Curricular festgelegte Lehrpläne würden der Vielfalt der Lernenden nicht gerecht und es bedürfe autonomer Bildungsräume, die durch demokratische Schulen optimal realisiert werden könnten [Ullrich, Strunck, 2012, s. 13]. Ein Großteil demokratischer Schulen, nach der Meinung von U. Klemm (2013), zeichnet sich durch folgende pädagogische Leitprinzipien aus:

- Es gibt keine standardisierten Lehrpläne und auch keine Noten. Inhalte, Ziele und Methoden werden von den Schülern selbst festgelegt und mithilfe von Lernbegleiter konzipiert und durchgeführt; 
- In demokratischen Schulen ist Lernen verbunden mit Entdecken, Experimentieren und Spielen; nicht aber mit Reglementierung, Anleitung und Drill;

- Die Lehrer - Schüler - Hierarchie wird radikal aufgehoben. Stattdessen lernen die Beteiligten kooperativ und gleichberechtigt mit anderen;

- Das Lernen beschränkt sich auf den Lernort Schule. Dies ist mit einer Entgrenzung des Klassenzimmers verbunden, um alternative Lehrerfahrungen in außerschulischen Lernorten sammeln zu können;

- Lernprozesse und Lernziele ergeben sich aus den Alltagserfahrungen. Jeder Schüler bestimmt individuell nach Zeit, Umfang und Tempo die eigenen Lernfortschritte;

- Demokratische Schulen sind inklusive Lern- und Lebensorte. Alle Kinder, Jugendlichen und Erwachsenen haben hier das gleiche Recht auf Selbstbestimmung und Schutz. Die Bedürfnisse aller Beteiligten werden gleichermaßen geachtet;

- In regelmäßigen Kinder-/Schulversammlungen haben alle Schüler und Mitarbeiter die Möglichkeit, Einfluss auf die Struktur und Organisation der Schule zu nehmen. Schüler und Mitarbeiter sind gleichberechtigt in Entscheidungsprozesse eingebunden und müssen sich gleichermaßen an die Schulregeln halten. Bei Regelverstößen werden alle Beteiligten in gleicher Weise zur Verantwortung gezogen;

- Gelernt wird in altersgemischten Gruppen unter der Prämisse, dass Kinder unterschiedlicher Entwicklungsstufen voneinander lernen können [Klemm, 2013, s. 119].

Wie schön Bekannt wurde, es ist zu beachten, dass diese Kriterien nicht in gleicher Form für alle demokratischen Schulen gelten. Die Konzepte der Schulen unterschieden sich teilweise deutlich voneinander. Detaillierte Darstellungen sind jedoch im Rahmen dieser Arbeit nicht sinnvoll, weil es um die grundlegenden Ideen gehen soll. Nach Schätzungen der European Democratic Education Community gibt es derzeit mehr als 70 demokratische Schulen in Europa und viele weitere Schulen Weltweit [EDUEC-Mietgliedschulen, 2015]. Inhaltlich beziehen sich demokratische Schulen auf die Konzepte und Ansätze von Reformpädagogen, wie z.B. Jean-Jacques Rousseau, Celestin Freinet, Maria Montessori, Humberto Maturana, John Dewey, Jean Piaget, Jesper Juul, Carl Rogers [Wiesemann, Amman, 2012, s. 103].

Als sehr wichtige Initiative im Kontext Sozialer Arbeit und Inklusion ist gezielt aktiver Umgang mit künstlerischen Ausdrucksformen, kreativen Prakti- 
ken und sinnlichen Erfahrungen zu verstehen. Künstlerische Ausdrucksformen sind seit Jahren fester Bestandteil Sozialer Arbeit. Die Perspektiven Kontinuierlich änderten sich mit einer lebensweltorientierten Sozialarbeit, die die ganzheitliche Betrachtung des Alltags zum Gegenstand hat. Es entwickeln sich stadtteil- und zielgruppenspezifische Kulturangebote, die nicht nur eine Freizeitbeschäftigung ermöglichen wollen. Hier geht es vor allem um eine künstlerisch-kritische Auseinandersetzung mit den Alltagsrealitäten und Problemlagen der Adressaten. In heutigen Zeit Kulturelle Bildung in der Sozialen Arbeit in Kontexts Inklusion Prozess will nicht Bestandteil von Elitebildung sein und vielmehr als Teil einer „politischen Bewegung von unten“, die grundsätzlich allen Menschen Zugänge zur künstlerischen Praxis ermöglichen soll. Wie die Wissenschaftler und Pädagogen behaupten künstlerisch-ästhetische Methoden in der Sozial Arbeiten sollen Selbsthilfe und Selbstbildungspotenziale aktivieren, um Menschen mit Beeinträchtigungen zu befähigen, ein möglichst selbstbestimmtes und autonomes Leben führen zu können. Wie die Forscher M.S. Meis und G.A. Mies (2012) behaupten, ziele die künstlerisch-ästhetisch Praxis in der Sozialen Arbeit ab auf: „Selbststeuerung; Generierung; Aufarbeitung und Vernetzung eigener Erlebnisse und Erfahrungen; Differenzierung von Wahrnehmung; neue Eindrücke und neue Eindrucksformen; Kommunikation und Interaktion; (eigen-sinnigen) Erwerb von Wissen, Kenntnissen und Fertigkeiten; selbständiges Forschen/Erforschen von alltagsrelevanten Zusammenhängen; (Welt-) Erfahrung und (Welt-) Zugang; schöpferisch-produktiv sein, Werte schaffen und Sinn stiften; Lebensfreude und Glück; gesellschaftliche und kulturelle Teilhabe“ [Meis, Mies, 2012, s. 39].

Inklusion wird nicht nur im schulischen Kontext diskutiert und geforscht, sondern ist auch in den Theoriediskuskursen der Sozialen Arbeit prominent vertreten. Einen zentralen Stellenwert nehmen dabei der Approach und systemtheoretische Perspektiven ein, die im gesellschaftsanalytisches Potenzial hin untersucht sind und weiter geforscht seien sollen. Das bedeutet, dass um die Vorstellung des guten Lebens zu konkretisieren, formuliert M. Nussbaum (1999) eine Liste von zehn Basisfähigkeiten, die als wesentliche Erfordernisse eines menschenwürdigen Lebens angesehen werden. Zu diesen Fähigkeiten Autorin zählt:

1. „Die Fähigkeit, ein volles Menschenleben bis zum Ende zu führen; nicht vorzeitig zu sterben oder zu sterben, bevor das Leben so reduziert ist, dass es nicht mehr lebenswert ist; 
2. Die Fähigkeit, sich guter Gesundheit zu erfreuen: sich angemessen zu ernähren; eine angemessene Unterkunft zu haben; Möglichkeiten zu sexueller Befriedigung zu haben; sich von einem Ort zu einem anderen zu bewegen;

3. Die Fähigkeit, unnötigen Schmerz zu vermeiden und friedvolle Erlebnisse zu haben;

4. Die Fähigkeit, die fünf Sinne zu benutzen, sich etwas vorzustellen, zu denken und zu urteilen;

5. Die Fähigkeit, Bindungen zu Dingen und Personen außerhalb unser selbst zu haben; diejenigen zu lieben, die uns lieben und für uns sorgen, und über ihre Abwesenheit traurig zu sein; allgemein gesagt: zu lieben, zu trauern, Sehnsucht und Dankbarkeit zu empfinden;

6. Die Fähigkeit, sich eine Vorstellung vom Guten zu machen und kritisch über die eigene Lebensplanung nachzudenken;

7. Die Fähigkeit für andere und bezogen auf andere zu leben, Verbundenheit mit anderen Menschen zu erkennen und zu zeigen; verschiedene Formen von familiären und sozialen Beziehungen einzugehen;

8. Die Fähigkeit, in Verbundenheit mit Tieren, Pflanzen und der ganzen Natur zu leben und pfleglich mit innen umzugehen;

9. Die Fähigkeit, zu lachen, zu spielen und Freude an erholsamen Tätigkeiten zu haben;

10. Die Fähigkeit, sein eigenes Leben und nicht das von jemand anderem zu leben" [Nussbaum, 1999, s. 57ff.].

Anknüpfen an die obengenannten Überlegungen und theoretischpraktischen Ideen, man kann zusammenfassend sagen, dass alle Wissenschaftler, Sonderpädagogen und Therapeuten in ihrem geführten Initiativen zur sozialen Eingliederungen und Forschungen zur Verbesserung der Lebensqualität von Menschen mit Behinderungen in Deutschland einig sind, dass im Inklusionsdiskurs werden vor allem Postulate für eine gelungene Inklusion entwickelt, während die Erforschung der Gründe des Scheiterns inklusiver Ansätze vergleichsweise wenig Beachtung erfährt. Trotzdem, das Standarden werden weiter hin mit verschiedenen inklusiven Verpflichtungen und Konsequenzen Theoretisch und zusammen mit Praxis verknüpft. Das soll bedeutet, dass auch Menschen mit Behinderung profitieren sollen davon, wenn sie nicht mehr im vereinfachten Rangvergleich bewertet werden, sondern ihre 
individuellen Stärken auf der Basis gemeinsamer Grundqualifikationen entfalten können.

\section{Literatur}

Amrhein B., (2001), Inklusion in der Sekundarstufe. Eine empirische Analyse, Verlag Julius Klinkhardt, Bad Heilbrunn.

Boban I., Hinz A., (2010), Was Inklusion ist und was nicht, [In:] Die Grundschulzeitschrift 240/2010.

Boban I., Hinz A., (2012), Individuelle Förderung in der Grundschule? Spannungsfelder und Perspektiven im Kontext inklusiver Pädagogik und demokratischer Bildung, [In:] C. Solzbacher, S. Müller-Using, I. Doll (Hrsg.), Ressourcen stärken! Individuelle Förderung für die Grundschule, Wolters Kluwer, Köln.

Boban I., Hinz A., Plate E., Tiedeken P., (2014), Inklusion in Worte fassen - eine Sprache ohne Kategorisierungen? [In:] S. Schuppener, N. Bernhardt, M. Hauser, F. Poppe (Hrsg.), Inklusion und Chancengleichheit. Diversity im Spiegel von Bildung und Didaktik, Klinkhardt, Bad Heilbrunn.

Bundesministerium für Arbeit und Soziales (2011), Übereinkommen der Vereinten Nationen über die Rechte von Menschen mit Behinderungen, Berlin.

Bundesministerium für Arbeit und Soziales (2013), Teilhabebericht der Bundesregierung über die Lebenslagen von Menschen mit Beeinträchtigungen, Teilhabe-Beeinträchtigung-Behinderung, Berlin.

Dannenbeck C., (2015), Inklusion und ästhetische Praxis. Herausforderung für die professionelle Praxis, [In:] T. Grosse, L. Niederreiter, H. Skladny (Hrsg.), Inklusion und Ästhetische Praxis in der Sozialen Arbeit, Weinheim und Basel, Belz Juventa.

Dorrance C., Mayberg W., (2015), Inklusion spielerisch auf den Punkt gebracht: Txalaparta - ein Rhythmusinstrument aus dem Baskenland, [In:] T. Grosse, L. Niederreiter, H. Skladny (Hrsg.), Inklusion und Ästhetische Praxis in Sozialen Arbeit, Beltz Juventa, Weinheim und Basel.

Eberwein H., Mand J. (Hrsg.), (2008), Integration konkret. Begründung, didaktische Konzepte, inklusive Praxis, Bad Heilbrunn, Verlag Julius Klinkhardt.

European Democratic Education Community (2015), EDUEC-Mietgliedschulen. Online abrufbar unter: http://www.eudec.org [15.03.2019].

Hinz A., (2012), Inklusion - historische Entwicklungslinien und internationale Kontexte, [In:] A. Hinz, I. Körner, U. Niehoff (Hrsg.), Von der Integration zur Inklusion, Grundlagen - Perspektiven - Praxis, Lebenshilfe Verlag, 3, Marburg. 
Hinz A., Körner I., Niehoff U. (2012), Von der Integration zur Inklusion, Grundlagen - Perspektiven - Praxis, Lebenshilfe-Verlag, Marburg.

Kast J.I., (2017), Einführung in die Soziologie der Behinderung, Springer Fachmedien, Wiesbaden.

Klemm K., Preuss-Lausitz U., (2011), Auf dem Weg zur schulischen Inklusion in Nordrhein-Westfalen. Empfehlungen zur Umsetzung der UN-Behindertenrechtskonvention im Bereich der allgemeinen Schulen, Nordrhein-Westfalen.

Klemm U., (2013), Geschichte und Gegenwart Freier Demokratischer Schulen. Warte, bis der Schüler den ersten Schritt macht, [In:] A. Gürlevik, Ch. Palentien, R. Heyer (Hrsg.), Privatschulen versus staatliche Schulen, VS Verlag für Sozialwissenschaften, Wiesbaden.

Kröger F., Merkt I., Sievers N., (2014), Inklusive Kulturelle Bildung und Kulturarbeit, Förderer und Akteure - Programme und Projekte, Institut für Kulturpolitik (IfK) der Kulturpolitischen Gesellschaft e.V., Bonn.

Meis M.S., Mies G.A. (Hrsg.), (2012), Künstlerisch-ästhetische Methoden in der Sozialen Arbeit: Kunst, Musik, Theater, Tanz und Neue Medien, Kohlhammer, Stuttgart.

Niedersächsisches Ministerium für Soziales, (2017), Aktions-Plan Inklusion Niedersachsen für die Jahre 2017 und 2018 in Leichter Sprache, Gesundheit und Gleichstellung, Hannover.

Nussbaum M., (1999), Gerechtigkeit oder das gute Leben, Suhrkamp, Frankfurt am Main.

Sauer K.E., (2014), Inklusion aus jugendkultureller Perspektive. Wege der Kommunikation in Musikprojekten von Jugendlichen verschiedener Herkunft mit unterschiedlichen Lernvoraussetzungen, Centaurus, Herbolzheim.

Ullrich H., Strunck S., (2012), Private Schulen in Deutschland. Entwicklungen und Diskurse, [In:] H. Ullrich, S. Strunck (Hrsg.), Privaten Schulen in Deutschland. Entwicklung - Profile - Kontroversen, VS Verlag für Sozialwissenschaften, Wiesbaden.

Wiesemann J., Amman K., (2012), Freie Alternativschulen, [In:] H. Ullrich, S. Strunck (Hrsg.), Privaten Schulen in Deutschland. Entwicklungen - Profile - Kontroversen. VS Verlag für Sozialwissenschaften, Wiesbaden. 